\title{
304 New Table of Heights and Weights
}

\section{NEW TABLE OF HEIGHTS AND WEIGHTS}

To the Editor of the Transactions of the Faculty of Actuaries.

DeAR SIR,

Heights and Weights.

We enclose a print of a new Table which has been deduced from the experience of seven British Life Offices, and also a copy of an abridged summary including comparative figures taken from the two other sources specified. You will observe from this latter document that, although the new weights are almost always somewhat less than those shown by the other investigations, the differences are slight except for the taller entrants. There appears to be no doubt that this feature is inherent in the data and is not a result of the graduation. We may add that the unadjusted data for the various ages at entry were collected into groups of five, and that the rough average weights deduced from these grouped figures were subjected to a process of graphic graduation in order to obtain the final values. As a matter of fact the original data needed very little adjustment except at the upper and lower limits of height.

Possibly you may consider the results to be of sufficient interest to warrant publication in the Transactions of the Faculty.

Yours faithfully,

A LEVINE, Actuary.

aldiance assorance Company, Limited,

LoNDon, 2nd July 1923. 


\section{Hetghts AND Whights.}

[MALE LIVES]

$A$ Table deduced from the records of seven Life Offices in respect of 28,697 Medical Examinations for Assurance made during the years 1921 and 1922.

\begin{tabular}{|c|c|c|c|c|c|c|c|c|c|c|c|c|c|c|c|c|}
\hline \multirow{2}{*}{ 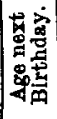 } & \multicolumn{15}{|c|}{ HELGHT. } & \multirow{2}{*}{ 若 } \\
\hline & $\begin{array}{ll}5^{\prime} & 3^{\prime \prime}\end{array}$ & $5^{\prime} \quad 4^{\prime \prime}$ & $5^{\prime}$ & $5^{\prime \prime}$ & $5^{\prime} \quad 6^{\prime \prime}$ & $5^{\prime}$ & & $5^{\prime} \quad 8^{\prime \prime}$ & $\left|\begin{array}{ll}E^{\prime} & 9^{\prime \prime}\end{array}\right|$ & $5^{\prime} 10^{\prime \prime}$ & $5^{\prime} 11^{\prime \prime}$ & $\left|\begin{array}{ll}6^{\prime} & 0^{\prime \prime}\end{array}\right|$ & $6^{\prime} 1^{\prime \prime}$ & $6^{\prime \prime}$ & $\mathbf{3}^{\prime} \mathbf{2}^{\prime \prime}$ & \\
\hline $\begin{array}{l}15 \\
16 \\
17 \\
18 \\
19\end{array}$ & $\begin{array}{rr}\text { st. } & \text { lbs. } \\
8 & 2 \\
8 & 3 \\
8 & 5 \\
8 & 6 \\
8 & 7\end{array}$ & $\begin{array}{rr}\text { st. } & \text { lbs. } \\
8 & 6 \\
8 & 7 \\
8 & 8 \\
8 & 9 \\
8 & 11\end{array}$ & $\begin{array}{r}\text { st. } \\
8 \\
8 \\
8 \\
8 \\
9\end{array}$ & $\begin{array}{l}10 \\
12 \\
13\end{array}$ & $\mid \begin{array}{rr}\text { st. } & \text { lbs. } \\
8 & 12 \\
8 & 13 \\
9 & 1 \\
9 & 2 \\
9 & 3\end{array}$ & \begin{tabular}{|l} 
st. ll \\
9 \\
9 \\
9 \\
9 \\
9
\end{tabular} & \begin{tabular}{r|r} 
bs. & s \\
1 & \\
3 & \\
4 \\
5 \\
7
\end{tabular} & \begin{tabular}{|rr} 
st. & lbs. \\
9 & 5 \\
9 & 6 \\
9 & 8 \\
9 & 9 \\
9 & 11
\end{tabular} & $\left|\begin{array}{rr}\text { st. } & \text { lbs. } \\
9 & 9 \\
9 & 10 \\
9 & 11 \\
9 & 13 \\
10 & 0\end{array}\right|$ & $\begin{array}{rr}\text { st. } & \text { lbs. } \\
9 & 12 \\
10 & 0 \\
10 & 1 \\
10 & 3 \\
10 & 4\end{array}$ & $\begin{cases}\text { st. } & \text { lbs } \\
10 & 2 \\
10 & 4 \\
10 & 5 \\
10 & 6 \\
10 & 8\end{cases}$ & $\left|\begin{array}{lr}\text { st. } & \text { lbs. } \\
10 & 6 \\
10 & 8 \\
10 & 9 \\
10 & 11 \\
10 & 12\end{array}\right|$ & $\begin{array}{lr}\text { st. } & \text { lbs } \\
10 & 10 \\
10 & 12 \\
10 & 13 \\
11 & 1 \\
11 & 2\end{array}$ & \begin{tabular}{l|l} 
s. & st. \\
0 & 1 \\
2 & 1. \\
3 & 1 \\
1 & 1. \\
2 & 1.
\end{tabular} & $\begin{array}{cc}\text { t. los. } \\
11 & 0 \\
1 & 2 \\
1 & 3 \\
1 & 5 \\
11 & 6\end{array}$ & $\begin{array}{l}15 \\
16 \\
17 \\
18 \\
19\end{array}$ \\
\hline $\begin{array}{l}20 \\
21 \\
22 \\
23 \\
24\end{array}$ & $\begin{array}{rr}8 & 9 \\
8 & 10 \\
8 & 11 \\
8 & 12 \\
8 & 13\end{array}$ & $\begin{array}{rr}8 & 12 \\
8 & 13 \\
9 & 0 \\
9 & 1 \\
9 & 2\end{array}$ & & & $\begin{array}{ll}9 & 5 \\
9 & 6 \\
9 & 7 \\
9 & 8 \\
9 & 9\end{array}$ & $\begin{array}{l}9 \\
9 \\
9 \\
9 \\
9 \\
9 \\
1\end{array}$ & $\begin{array}{r}8 \\
9 \\
11 \\
12 \\
13\end{array}$ & $\begin{array}{rr}9 & 12 \\
9 & 13 \\
10 & 0 \\
10 & 1 \\
10 & 2\end{array}$ & $\left|\begin{array}{ll}10 & 2 \\
10 & 3 \\
10 & 4 \\
10 & 5 \\
10 & 6\end{array}\right|$ & $\begin{array}{rr}10 & 5 \\
10 & 6 \\
10 & 8 \\
10 & 9 \\
10 & 10\end{array}$ & $\begin{array}{rr}10 & 9 \\
10 & 11 \\
10 & 12 \\
10 & 13 \\
11 & 0\end{array}$ & $\left|\begin{array}{rr}10 & 13 \\
11 & 1 \\
11 & 2 \\
11 & 3 \\
11 & 4\end{array}\right|$ & $\mid \begin{array}{ll}11 & 3 \\
11 & 4 \\
11 & 6 \\
11 & 7 \\
11 & 8\end{array}$ & \begin{tabular}{l|l}
3 & 1 \\
4 & 1 \\
6 & 1 \\
7 & 1. \\
8 & 1.
\end{tabular} & $\begin{array}{rr}1 & 7 \\
11 & 9 \\
1 & 10 \\
1 & 11 \\
1 & 12\end{array}$ & $\begin{array}{l}20 \\
21 \\
22 \\
23 \\
24\end{array}$ \\
\hline $\begin{array}{l}25 \\
26 \\
27 \\
28 \\
29\end{array}$ & $\begin{array}{ll}9 & 0 \\
9 & 1 \\
9 & 2 \\
9 & 3 \\
9 & 4\end{array}$ & $\begin{array}{ll}9 & 3 \\
9 & 4 \\
9 & 5 \\
9 & 6 \\
9 & 7\end{array}$ & & $\begin{array}{r}7 \\
8 \\
9 \\
10 \\
11\end{array}$ & $\mid \begin{array}{rr}9 & 10 \\
9 & 11 \\
9 & 12 \\
9 & 13 \\
10 & 0\end{array}$ & $\begin{array}{l}10 \\
10 \\
10 \\
10 \\
10\end{array}$ & $\begin{array}{l}0 \\
1 \\
1 \\
2 \\
3\end{array}$ & $\begin{array}{ll}10 & 3 \\
10 & 4 \\
10 & 5 \\
10 & 6 \\
10 & 7\end{array}$ & $\mid \begin{array}{rr}10 & 7 \\
10 & 8 \\
10 & 9 \\
10 & 9 \\
10 & 10\end{array}$ & $\begin{array}{ll}10 & 11 \\
10 & 12 \\
10 & 12 \\
10 & 13 \\
11 & 0\end{array}$ & $\begin{array}{ll}11 & 1 \\
11 & 2 \\
11 & 3 \\
11 & 4 \\
11 & 5\end{array}$ & $\left|\begin{array}{ll}11 & 5 \\
11 & 6 \\
11 & 7 \\
11 & 8 \\
11 & 9\end{array}\right|$ & $\begin{array}{rr}11 & 9 \\
11 & 10 \\
11 & 11 \\
11 & 12 \\
11 & 13\end{array}$ & \begin{tabular}{l|l}
9 & 1 \\
0 & 1 \\
1 & 1 \\
2 & 1 \\
3 & 1
\end{tabular} & $\begin{array}{ll}12 & 0 \\
12 & 1 \\
12 & 3 \\
2 & 4\end{array}$ & $\begin{array}{l}25 \\
26 \\
27 \\
28 \\
29\end{array}$ \\
\hline $\begin{array}{l}30 \\
3 \mathrm{I} \\
32 \\
33 \\
34\end{array}$ & $\begin{array}{ll}9 & 5 \\
9 & 5 \\
9 & 6 \\
9 & 6 \\
9 & 7\end{array}$ & $\begin{array}{rr}9 & 8 \\
9 & 9 \\
9 & 9 \\
9 & 10 \\
9 & 10\end{array}$ & $\begin{array}{r}9 \\
9 \\
9 \\
9 \\
10\end{array}$ & $\begin{array}{r}11 \\
12 \\
13 \\
13 \\
0\end{array}$ & $\mid \begin{array}{ll}10 & 1 \\
10 & 1 \\
10 & 2 \\
10 & 3 \\
10 & 3\end{array}$ & $\begin{array}{l}10 \\
10 \\
10 \\
10 \\
10\end{array}$ & $\begin{array}{l}4 \\
5 \\
5 \\
6 \\
7\end{array}$ & $\begin{array}{ll}10 & 8 \\
10 & 9 \\
10 & 10 \\
10 & 10 \\
10 & 11\end{array}$ & $\mid \begin{array}{rr}10 & 11 \\
10 & 12 \\
10 & 13 \\
10 & 13 \\
11 & 0\end{array}$ & $\begin{array}{ll}11 & 1 \\
11 & 2 \\
11 & 2 \\
11 & 3 \\
11 & 4\end{array}$ & $\begin{array}{ll}11 & 5 \\
11 & 6 \\
11 & 7 \\
11 & 8 \\
11 & 9\end{array}$ & $\mid \begin{array}{ll}11 & 10 \\
11 & 11 \\
11 & 12 \\
11 & 12 \\
11 & 13\end{array}$ & $\mid \begin{array}{ll}12 & 0 \\
12 & 1 \\
12 & 2 \\
12 & 3 \\
12 & 4\end{array}$ & $\begin{array}{ll}0 & 1 \\
1 & 1 \\
2 & 1 \\
3 & 1 \\
4 & 1\end{array}$ & $\begin{array}{ll}12 & 5 \\
12 & 6 \\
12 & 7 \\
12 & 8 \\
12 & 9\end{array}$ & $\begin{array}{l}30 \\
31 \\
32 \\
33 \\
34\end{array}$ \\
\hline $\begin{array}{l}35 \\
36 \\
37 \\
38 \\
39\end{array}$ & $\begin{array}{ll}9 & 7 \\
9 & 8 \\
9 & 8 \\
9 & 9 \\
9 & 9\end{array}$ & $\begin{array}{ll}9 & 11 \\
9 & 11 \\
9 & 12 \\
9 & 12 \\
9 & 13\end{array}$ & $\begin{array}{l}10 \\
10 \\
10 \\
10 \\
10\end{array}$ & & $\begin{array}{ll}10 & 4 \\
10 & 4 \\
10 & 5 \\
10 & 6 \\
10 & 6\end{array}$ & $\begin{array}{l}10 \\
10 \\
10 \\
10 \\
10\end{array}$ & $\begin{array}{r}8 \\
8 \\
9 \\
10 \\
10\end{array}$ & $\mid \begin{array}{rr}10 & 12 \\
10 & 12 \\
10 & 13 \\
11 & 0 \\
11 & 0\end{array}$ & $\left|\begin{array}{ll}11 & 1 \\
11 & 2 \\
11 & 2 \\
11 & 3 \\
11 & 4\end{array}\right|$ & $\begin{cases}11 & 4 \\
11 & 5 \\
11 & 6 \\
11 & 7 \\
11 & 8\end{cases}$ & $\left\{\begin{array}{rr}11 & 9 \\
11 & 10 \\
11 & 11 \\
11 & 12 \\
11 & 13\end{array}\right.$ & $\begin{array}{ll}12 & 0 \\
12 & 1 \\
12 & 2 \\
12 & 3 \\
12 & 3\end{array} \mid$ & $\begin{array}{ll}12 & 5 \\
12 & 6 \\
12 & 7 \\
12 & 8 \\
12 & 8\end{array}$ & \begin{tabular}{l|l}
5 & 1 \\
6 & 1 \\
7 & 1 \\
8 & 1 \\
8 & 1
\end{tabular} & & $\begin{array}{l}35 \\
36 \\
37 \\
38 \\
39\end{array}$ \\
\hline $\begin{array}{l}40 \\
4 I \\
42 \\
43 \\
44\end{array}$ & $\begin{array}{ll}9 & 10 \\
9 & 10 \\
9 & 10 \\
9 & 11 \\
9 & 11\end{array}$ & $\mid \begin{array}{rr}9 & 13 \\
10 & 0 \\
10 & 0 \\
10 & 1 \\
10 & 1\end{array}$ & $\begin{array}{l}10 \\
10 \\
10 \\
10 \\
10\end{array}$ & $\begin{array}{l}3 \\
4 \\
4 \\
4 \\
5\end{array}$ & $\begin{array}{ll}10 & 7 \\
10 & 7 \\
10 & 8 \\
10 & 8 \\
10 & 9\end{array}$ & \begin{tabular}{l|l}
7 & 10 \\
8 & 10 \\
8 & 10 \\
8 & 10 \\
9 & 10
\end{tabular} & & $\begin{array}{ll}11 & 1 \\
11 & 2 \\
11 & 2 \\
11 & 3 \\
11 & 3\end{array}$ & $\mid \begin{array}{ll}11 & 5 \\
11 & 5 \\
11 & 6 \\
11 & 6 \\
11 & 7\end{array}$ & $\mid \begin{array}{rr}11 & 9 \\
11 & 9 \\
11 & 10 \\
11 & 11 \\
11 & 12\end{array}$ & $\begin{cases}12 & 0 \\
12 & 0 \\
12 & 1 \\
12 & 2 \\
12 & 2\end{cases}$ & $\begin{array}{ll}0 \\
12 & 4 \\
12 & 5 \\
12 & 6 \\
12 & 6 \\
12 & 7\end{array}$ & $\begin{array}{rr}12 & 9 \\
12 & 10 \\
12 & 10 \\
12 & 11 \\
12 & 12\end{array}$ & \begin{tabular}{l|l}
9 & 1 \\
0 & 1 \\
0 & 1 \\
1 & 1 \\
2 & 1
\end{tabular} & $\begin{array}{ll}13 & 0 \\
13 & 0 \\
13 & 1 \\
13 & 1 \\
13 & 2\end{array}$ & $\begin{array}{l}40 \\
4 I \\
42 \\
43 \\
44\end{array}$ \\
\hline $\begin{array}{l}45 \\
46 \\
47 \\
48 \\
49\end{array}$ & $\begin{array}{ll}9 & 12 \\
9 & 12 \\
9 & 12 \\
9 & 12 \\
9 & 12\end{array}$ & $\begin{array}{l}10 \\
10 \\
10 \\
10 \\
10\end{array}$ & $\begin{array}{l}10 \\
10 \\
20 \\
10 \\
10\end{array}$ & $\begin{array}{l}5 \\
6 \\
6 \\
6 \\
7\end{array}$ & $\begin{array}{rr}10 & 9 \\
10 & 10 \\
10 & 10 \\
10 & 10 \\
10 & 11\end{array}$ & $\begin{array}{ll}9 & 10 \\
0 & 11 \\
0 & 11 \\
0 & 11 \\
1 & 11\end{array}$ & $\begin{array}{r}13 \\
0 \\
0 \\
1 \\
1\end{array}$ & $\begin{array}{ll}11 & 3 \\
11 & 4 \\
11 & 4 \\
11 & 5 \\
11 & 5\end{array}$ & $\left|\begin{array}{rr}11 & 8 \\
11 & 8 \\
11 & 9 \\
11 & 9 \\
11 & 10\end{array}\right|$ & $\left\{\begin{array}{rr}11 & 12 \\
11 & 12 \\
11 & 13 \\
12 & 0 \\
12 & 0\end{array}\right.$ & $\begin{array}{l}12 \\
12 \\
12 \\
12 \\
12\end{array}$ & \begin{tabular}{r|rr}
3 & 12 & 8 \\
3 & 12 & 8 \\
4 & 12 & 9 \\
5 & 12 & 10 \\
5 & 12 & 10
\end{tabular} & $\begin{array}{lr}12 & 12 \\
12 & 13 \\
12 & 13 \\
13 & 0 \\
13 & 0\end{array}$ & \begin{tabular}{l|l}
2 & 1 \\
3 & 1 \\
3 & 1 \\
0 & 1 \\
0 & 1
\end{tabular} & $\begin{array}{ll}13 & 3 \\
13 & 3 \\
13 & 4 \\
13 & 4 \\
13 & 5\end{array}$ & $\begin{array}{l}45 \\
46 \\
47 \\
48 \\
49\end{array}$ \\
\hline $\begin{array}{l}\mathbf{5 0} \\
\mathbf{5 1} \\
\mathbf{5 2} \\
\mathbf{5 3} \\
\mathbf{5 4}\end{array}$ & $\begin{array}{rr}9 & 13 \\
9 & 13 \\
9 & 13 \\
10 & 0 \\
10 & 0\end{array}$ & $\begin{cases}10 & 3 \\
10 & 4 \\
10 & 4 \\
10 & 4 \\
10 & 4\end{cases}$ & $\begin{array}{ll}3 & 10 \\
4 & 10 \\
4 & 10 \\
4 & 10 \\
4 & 10\end{array}$ & $\begin{array}{l}7 \\
8 \\
8 \\
9 \\
9\end{array}$ & $\mid \begin{array}{ll}10 & 11 \\
10 & 11 \\
10 & 12 \\
10 & 12 \\
10 & 12\end{array}$ & \begin{tabular}{l|l}
1 & 11 \\
1 & 11 \\
2 & 11 \\
2 & 11 \\
2 & 11
\end{tabular} & $\begin{array}{l}1 \\
2 \\
2 \\
2 \\
3\end{array}$ & $\begin{array}{ll}11 & 6 \\
11 & 6 \\
11 & 6 \\
11 & 7 \\
11 & 7\end{array}$ & $\mid \begin{array}{ll}11 & 10 \\
11 & 11 \\
11 & 11 \\
11 & 11 \\
11 & 12\end{array}$ & $\begin{array}{ll}12 & 1 \\
12 & 1 \\
12 & 2 \\
12 & 2 \\
12 & 3\end{array}$ & \begin{tabular}{l|l}
1 & 12 \\
12 \\
2 \\
2 \\
12 \\
12 \\
12 \\
12
\end{tabular} & $\begin{array}{lll}6 & 12 & 11 \\
6 & 12 & 11 \\
6 & 12 & 11 \\
7 & 12 & 12 \\
7 & 12 & 12\end{array}$ & $\begin{array}{ll}13 & 1 \\
13 & 1 \\
13 & 2 \\
13 & 2 \\
13 & 2\end{array}$ & \begin{tabular}{l|l}
1 & 1 \\
1 & 1 \\
2 & 1 \\
2 & 1 \\
2 & 1
\end{tabular} & $\begin{array}{ll}13 & 5 \\
13 & 6 \\
13 & 6 \\
13 & 6 \\
13 & 7\end{array}$ & $\begin{array}{l}50 \\
51 \\
52 \\
53 \\
54\end{array}$ \\
\hline $\begin{array}{l}\mathbf{5 5} \\
\mathbf{5 6} \\
\mathbf{5 7} \\
\mathbf{5 8} \\
\mathbf{5 9}\end{array}$ & $\begin{array}{ll}10 & 0 \\
10 & 0 \\
10 & 0 \\
10 & 0 \\
10 & 0\end{array}$ & $\begin{cases}10 & 4 \\
10 & 5 \\
10 & 5 \\
10 & 5 \\
10 & 5\end{cases}$ & \begin{tabular}{l|l}
4 & 10 \\
5 & 10 \\
5 & 10 \\
5 & 10 \\
5 & 10
\end{tabular} & $\begin{array}{l}9 \\
9 \\
9 \\
9 \\
9\end{array}$ & $\begin{array}{ll}10 & 12 \\
10 & 13 \\
10 & 13 \\
10 & 13 \\
10 & 13\end{array}$ & $\begin{array}{ll}2 & 11 \\
3 & 11 \\
3 & 11 \\
3 & 11 \\
3 & 11\end{array}$ & $\begin{array}{l}3 \\
3 \\
3 \\
3 \\
3\end{array}$ & $\begin{array}{ll}11 & 7 \\
11 & 8 \\
11 & 8 \\
11 & 8 \\
11 & 8\end{array}$ & $\left|\begin{array}{ll}11 & 12 \\
11 & 12 \\
11 & 12 \\
11 & 13 \\
11 & 13\end{array}\right|$ & $\begin{array}{ll}12 & 3 \\
12 & 3 \\
12 & 3 \\
12 & 3 \\
12 & 4\end{array}$ & \begin{tabular}{l|ll}
3 & 12 \\
3 & 12 \\
3 & 12 \\
3 & 12 \\
4 & 12 & \\
4
\end{tabular} & $\begin{array}{lll}7 & 12 & 12 \\
8 & 12 & 13 \\
8 & 12 & 13 \\
8 & 12 & 13 \\
8 & 12 & 13\end{array}$ & $\begin{array}{ll}13 & 3 \\
13 & 3 \\
13 & \\
13 \\
13\end{array}$ & \begin{tabular}{l|l}
3 & 1 \\
3 & 1 \\
3 & 1 \\
3 & 1 \\
4 & 1
\end{tabular} & $\begin{array}{ll}13 & 7 \\
13 & 7 \\
13 & 8 \\
13 & 8 \\
13 & 8\end{array}$ & $\begin{array}{l}55 \\
56 \\
57 \\
58 \\
59\end{array}$ \\
\hline 60 & 100 & 10 & 510 & 9 & 1013 & \begin{tabular}{l|l}
3 & 11
\end{tabular} & 3 & 118 & $\begin{array}{lll}11 & 13\end{array}$ & 124 & 4128 & \begin{tabular}{l|ll}
8 & 12 & 13
\end{tabular} & 13 & 41 & 138 & $\begin{array}{l}3 \\
60\end{array}$ \\
\hline
\end{tabular}




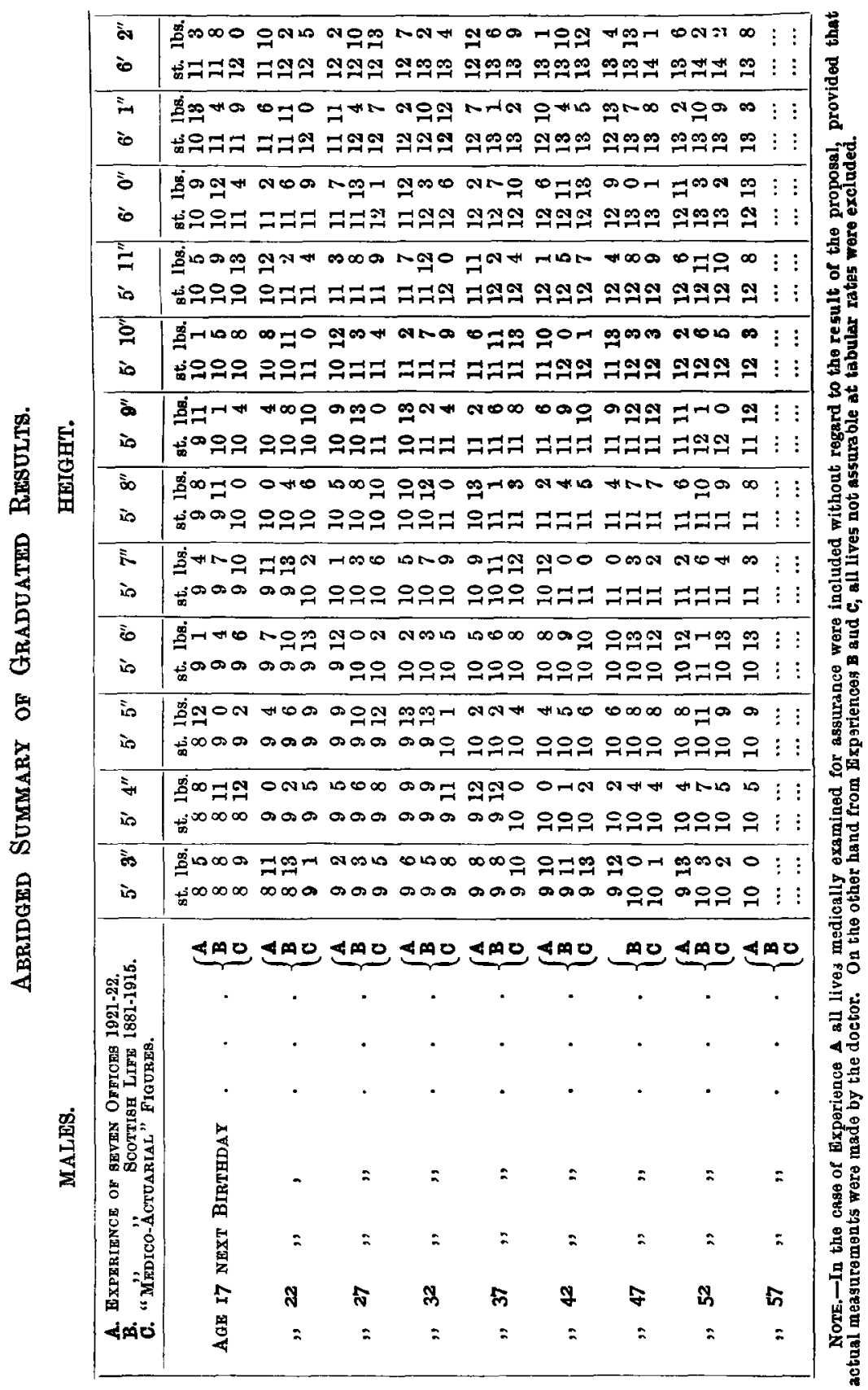

